JLL 6 (1)(2019)

\title{
L'ANALYSE DU DISCOURS CRITIQUE (ADC) DU CONFLIT ROHINGYA SUR LE JOURNAL LE MONDE
}

\author{
Ayudhia Ratna Wijaya ${ }^{\bowtie}$ Bernadus Wahyudi Joko Santoso \\ Departement de la Langue et la Littérature étrangere, Faculté des Langues et des Arts, \\ Universitas Negeri Semarang, Indonesia
}

\section{Info d'article}

Histoire de l'article:

Reçu fevriér 2019

Accepté avril 2019

Publié mai 2019

Mots-clés:

Discours Critique, Van Dijk,

Conflit Rohingya, Le Monde

\section{Extrait}

Les objectifs de cette recherche sont d'analyser a) la représentation ethnique de Rohingya et b) la représentation du gouvernement du Myanmar, des Nations Unies, de l'Union européenne, de l'Organisation de solidarité Rohingya, du Front Islamique Rohingya Arakan et du Bangladesh. Les approches de recherche utilisées sont l'approche méthodologique (descriptive qualitative) et celle de théorique (ADC van Dijk). Les quatre sources de données sont Le Monde (lemonde.fr), IndiaToday (indiatoday.com), BBC (bbc.com) et Editorial Media Indonesia (metrotvnews.com). Les données sont des mots, des syntagmes, des propositions, et des phrases contenant des éléments idéologiques, politiques, et sociaux. La méthode de recueillir des données est la méthode de lire attentivement avec la technique de base - la technique de recorder et la technique avancée - la technique de lire attentivement sans participation dans une interaction et la technique de copier-collerl. Les trois méthodes d'analyse des données sont la description, l'interprétation et l'explication. Pour présenter des résultats de l'analyse, j'utilise la méthode informelle. Après les avoir analysées, je trouve deux résultats : Le Monde présente le Rohingya, l'ONU, 1'UE, et le gouvernement du Bangladesh positivement, et au contraire Le Monde présente le RSO, l'ARIF et le gouvernement du Myanmar négativement. Les représentations montrées par Le Monde, IndiaToday, BBC, et Editorial Media Indonesia ont la relation très proche avec la cognition sociale qui sont historique, politique, idéologique, et émotif. Historiquement, ils sont nés pour présenter la liberté de diverses oppressions (l'oppression du gouvernement français, gouvernement indonésien, royaume anglais, et colonialisme britannique en Inde). Politiquement, ils supportent tous les programmes du gouvernement respectant les valeurs de 1'humanité et de la justice (centre-left/ centre-wing). Idéologiquement, ils respectent les valeurs des droits humanitaires et contrent tous les actions de génocides et de terroristes. Tandis que l'élément émotif se voit dans des utilisations des mots, des propositions, des phrases, des graphiques, et aussi des chronologies de discours (l'introduction, le contenu, et la clôture/la conclusion).

(C) 2019 Universitas Negeri Semarang 


\section{L'INTRODUCTION}

La langue est un moyen de communication parmi les individus. Parallèlement au développement de la linguistique dans les études appliquées, la langue s'est transformée en un instrument de pouvoir au sein du domaine multi disciplinaires. La langue est capable de fournir des informations explicites et implicites. Il se manifeste en faisant divers types de discours par différentes parties. Media est l'une des parties a un rôle majeur en tant qu'agent de diffusion d'informations. L'information est présentée sous la forme d'un discours qui a plusieurs objectifs. L'objectif principal est de maintenir la stabilité du pouvoir qui a été normalisé par l'information ou un message dans le discours. L'information implicite peut être comprise par le modèle d'analyse du discours critique. Il y a plusieurs types de modèles d'analyse de discours critique tels que; Fowler, van Leeuwen, Mills, van Dijk et Fairclough. Dans cette recherche, j'ai utilisé le modèle van Dijk dans l'analyse des discours. J'ai choisi le modèle de van Dijk parce qu'il est conformé au modèle d'analyse de discours médiatique qui est étroitement lié à la cognition des journalistes dans le processus de Production des discours. En outre, je me suis intéressé à discuter de l'analyse du discours critique, car je me suis intéressé à la science politique et sociale.

L'un des discours qui a attiré l'attention à beaucoup de gens dans Le Monde est le discours sur le conflit Rohingya qui a lieu au Myanmar. L'affaire du massacre des Rohingyas musulmans est considérée comme une violation de l'humanité comme le génocide à l'époque millénaire. Les militants politiques, les pionniers des droits de l'homme, les Nations Unies et même tous les citoyens du monde accordent une attention considérable à ce conflit. Le Monde, en tant que média le plus populaire en France, a participé activement au reportage sur le conflit Rohingya de 1991 à 2017.

Dans cette recherche, j'ai discuté du conflit Rohingya dans le journal Le Monde en 1991-2017 à travers l'analyse des éléments de la macrostructure, de la superstructure, de la microstructure et des éléments de la cognition sociale affectant les journalistes dans la production de discours du conflit Rohingya. Les résultats globaux de la recherche mèneront aux alignements de Le Monde en réponse au conflit Rohingya.

\section{LA THEORIE}

Cette section contient plusieurs théories. Ce sontla théorie de l'analyse du discours,l'analyse du discours critique, les caracteristiques de l'analyse du discours critique, le modèle d'analyse du discours critique de van Dijk, le discours comme lapratique sociale, le discours, l'idéologie et le hégémonie, et théorie de l'intertextualité.

\section{Analyse du discours}

L'analyse du discours reçoit des définitions tres variées. Il existe une définition très large, l'analyse de l'usage de la langue (Brown et Yule 1983 :1).Dubois (2001:150) dans le Dictionnaire de La Linguistique menyatakan bahwa "Le discours est une unité égale ou supérieure à la phrase; il est constitué par une suite formant un message ayant un commencement et une clôture."

\section{Analyse du discours critique}

L'analyse du discours critique (que nous abrégerons dorénavant en ADC) voit le langage comme une "pratique sociale " (Fairclough, 2001:18) et considère le contexte de l'utilisation du langage comme crucial (Wodak \& Fairclough, 1989 :21). L'ADC doit être définies comme fondamentalement intéressées à l'analyse des relations structurelles de domination, de discrimination, de pouvoir et de contrôle, telles qu'elles se manifestent dans le langage, qu'elles soient transparentes ou opaques. 


\section{Caractéristiques de l'analyse du discours critique}

Fairclough et Wodak, Jorgensen et Phillips (2007: 115-120) identifient cinq traits communs de l'analyse critique du discours.

1. La nature de la structure et les processus culturels et sociaux sont la langue du discours.

2. Le discours est une forme de pratique sociale qui constitue Le Monde social et se compose d'autres pratiques sociales.

3. L'utilisation du langage devrait être analysée empiriquement dans son contexte social.

4. Fonction de discours idéologiquement.

5. Recherche critique. L'analyse du discours critique ne peut pas être considérée comme une approche politiquement neutre, mais comme une approche critique qui est politiquement consacré à la montée du changement social. Au nom de l'émancipation, l'approche du discours critique favorise les groupes sociaux opprimés.

\section{Modèle d'analyse du discours critique van Dijk}

Van Dijk m'intéresse à la manière dont le pouvoir, et plus particulièrement l'abus de pouvoir est reproduit dans la société, et tout spécifiquement à la manière dont le discours est impliqué dans ce processus (van Dijk, 1988:1). Le point de départ de Van Dijk consiste à remettre en question l'automatisme théorique qui tend à rabattre la notion de contexte sur celle de situation. Si l'on se borne à définir le contexte comme la situation dans laquelle le discours advient, on se heurte immédiatement au problème de sa descriptibilité a priori par l'analyste. L'extrême complexité de toute situation fait courir à la description un risque bien réel : celui de ne jamais finir. On part le plus souvent du contexte " local " pour examiner les propriétés des participants et des circonstances spatio-temporelles immédiates, mais rien n'empêche que l'on étende ensuite la description à des contextes aussi "globaux ». Dans l'étude de van Dijk il y a trois étapes, y compris, macrostructure, superstructure et microstructure.

\section{Discours comme la pratique sociale}

La relation entre le langage et la société avec le concept de discours est ce qui fait la cohésion de l'ADC. La conception du discours s'organise tout le reste des emprunts théoriques, qu'ils soient philosophiques ou sociologiques, ou autres : ils interviennent en complément pour nourrir une conception du monde et de la société centrée autour du discours. Le discours est le point nodal par lequel l'ADClie société et matérialité linguistique (et sémiotique). C'est autour de lui qu'orbitent les autres concepts-clé de l'ADC, a savoir idéologie et pouvoir. C'est également sa conception qui justifie le type de pratique analytique prônée par l'ADC:

CDA sees discourse - language use in speech and writing - as a form of'social practice'. (Fairclough\&Wodak, 1989: 152)

l'ADC voit le discours - l'usage langagier a l'écrit et a l'oral — comme une forme de « pratique sociale $\gg$.

\section{Discours, idéologie et hégémonie}

Le concept de genre est celui qui permet a Fairclough de revenir a la problématique qui a toujours été la sienne, a savoir celle du changement dans le discours reflétant et/ou entrainant un changement social. Cela se fait précisément a travers les ordres du discours, et il rejoint sur ce point ses travaux précédents, mettant en relation ordres du discours et hégémonie (selon Gramsci, et a sa suite E. Laclau et C. Mouffe) :

L'un des aspects de cette mise en ordre est la domination : certaines manières de créer de la signification sont dominantes ou majoritaire dans un ordre du discours spécifique, tandis que 
d'autres sont marginales, ou en opposition, ou "alternatives". Par exemple, il y a probablement une manière dominante de conduire une consultation entre un patient et son médecin au Royaume-Uni, mais il n'y en a pas qu'une, et d'autres manières de faire peuvent être adoptées ou développées a plus ou moins grande échelle en opposition a la manière dominante. La manière dominante maintient probablement une distance sociale entre les médecins et les patients, ainsi que l'autorité du médecin sur la manière dont l'interaction doit procéder, mais il y a d'autres manières de faire qui sont plus "démocratiques", dans lesquelles les médecins n'abusent pas de leur autorité. Le concept politique d'hégémoniepeut être utile pour analyser les ordres du discours (Laclau\& Mouffe, 1985 ; Fairclough, 1992 ; Butler, et al. 2000). Une structuration particulière de la différence sémiotique peut devenir hégémonique, commencer a faire partie du sens commun qui sert a légitimer et a nourrir les relations de domination, mais l'hégémonie se verra toujours contester dans une proportion plus ou moins grande, par la lutte hégémonique. Un ordre du discours n'est pas un système clos ou rigide, c'est un système ouvert, qui est mis en danger par ce que ce passe réellement dans les interactions. [...] Même les discours puissants, comme les nouveaux discours de management, peuvent rencontrer des niveaux de résistance, ce qui a pour résultat qu'aucun des deux n'est mis en acte ou inculque a aucun degré. Lorsqu'on utilise unethéorie dialectique du discours, il faut prendre en compte, au cas par cas, les circonstances qui conditionnent si les entités sociales sont résistantesaux nouveaux discours, et à quel degré elles peuvent l'être. (Fairclough, 2001: 201)

Selon Norman Fairclough, les luttes et les tensions entre ordres du discours dominants et ordres du discours résistants résultent d'une perméabilité des genres. Les genres dominants vont coloniser d'autres genres, soit par association (genre chains (chaîne de genres)) soit par hybridation (genremixing (mélange de genres)).

Norman Fairclough tire sa définition de l'idéologie en grande partie des travaux de L. Althusser et de M. Pecheux, dont on trouve systématiquement les références bibliographiques dans les travaux qu'il publie dans les années 1980, preuve qu'il a été influence par le marxisme occidental et par l'AD française dans la première phase de ses recherches. Les premiers travaux de N. Fairclough essayaient de rendre compte de ce qu'il appelle le « changement socio-sémantique 》 (socio-sémantic change), c'estadire l'idée selon laquelle le changement social se traduit par un changement dans le discours et viceversa.

L'opération de l'idéologie va précisément être de déterminer ce que l'on peut penser, et par extension dire (id est les registres). En ce sens, les idéologies « sont des représentations partiales et qui induisent en erreur $\gg$ (Fairclough, 2001 [1989] : 134) :

L'opération de l'idéologie peut être vue comme une manière de construire des textes qui, de façon cumulative et constante, « imposent des vérités》aux interprètes de ces textes ainsi qu"a leurs producteurs, typiquement sans que personne ne s'en rende compte.L'influence du marxisme de L. Althusser se ressent également quand N. Fairclough definit le sens commun idéologique comme un « sens commun au service du maintien des relations de pouvoir inégales 》et de « l'établissement et la consolidation de la solidarité entre les membres de groupes sociaux particuliers 》 (Fairclough, 2001 [1989] : 70).

\section{Théorie de l'intertextualité}

La notion d'intertextualité est apparue à la fin des années 1960 au sein du groupe Tel Quel.Kristeva (1980:36-37) définit l'intertextualité comme une " interaction textuelle » qui permet de considérer « les différentes séquences (ou codes) d'une structure textuelle précise comme autant de transforms de séquences (codes) prises à d'autres textes. » Le texte littéraire se constituerait donc comme la transformation et la combinaison de différents textes antérieurs compris comme des codes utilisés par l'auteur. Elle montre ainsi que le roman médiéval Jehan de Saintré peut se définir comme 
l'interaction entre le texte de la scolastique, celui de la poésie courtoise, la littérature orale de la ville et le discours du carnaval.

\section{LA METHODOLOGIE DE LA RECHERCHE}

La source de données sont le discours sur le conflit Rohingya qui a été publié dans le journal en ligne Le Monde (lemonde.fr) en 1991-2017 et le discours sur le conflit Rohingya qui ont été publié dans IndiaToday (indiatoday.com), BBC (bbc.com) et Editorial Media Indonesia (metrotvnews.com). Les données sont des mots, des phrases ou des clauses qui contiennent des éléments politiques. La méthode de recueillir des données dans cette recherche est la méthode d'épargne (lire atttentivement) avec la technique de basse la technique de record et la technique avancée est la technique SBLC et Catat (technique de noter). La méthode d'analyse des données est la description, l'interprétation et l'explication.Jeprésente des résultats de l'analyse utilisant la méthode informelle.

\section{L'ANALYSE}

Cette sectioncontient quelques analyses. Ce sont l'analyse de macrostructure, l'analyse de superstructure, l'analyse microstructure et l'analyse de la représentation.

\section{Analyse macrostructurelle}

Basés sur les 16 discours analysés, il y a trois sujets de discussion. Ce sont la recherche de protectiondu Bangladesh par Rohingya, la perception du monde internationale sur le conflit Rohingya, et la compréhension du conflit Rohingya par Le Monde. Ils sont décrits dans la discussion suivante.

\section{Recherche de protectiondu Bangladesh par Rohingya}

Il y a trios discours qui ont été produit en 1991-1992. Ils ont le même sujet racontant la recherche de protectiondu Bangladesh par Rohingya. Ce sujet est justifié par les citations. Il y a trois citations justifiant ce sujet (ils se trouvent dans le chapitre 4 pages 70-72). L'une des citations est :

(1) Le discours de Sur la frontière du Bangladesh Une minorité réprimée 1991 a été produit par Le Monde.

«Des milliers de musulmans ont pour l'État d'Arakan, en Birmanie occidentale, pour se réfugier au Bangladesh,...»(Paragraphe 1) (Le Monde, Données 1)

La citation ci-dessus décrit la situation des Musulmans Rohingyas qui tente de fuir au Bangladesh. Ils essaient d'arriver au Bangladesh pour éviter la violence et l'oppression des militaires de la junte.

\section{Perception du monde internationale sur le conflit Rohingya}

Il y a 6 discours dans cette discussion qui se composent du discours «Nettoyage ethnique» en Birmanie chez les musulmans Rohingyas en 2000, celui des boat people Rohingyas Continent d'affluer en Indonésie et en Thailande en 2009, celui del'enfer sans frontières des Rohingyaen 2010, celui de Les Rohingya, celui d'un enfer l'autre en 2010, celui de Nouvelles exactions contre les Rohingyas en Birmanie en 2012, et celui deprésident birman à l'UE levée des sanctions en 2013. Les 6 discours ci-dessus ont le même sujet sur la perception internationale du conflit Rohingya. Il y a neuf citations justifiant ce sujet (ils se trouvent dans le chapitre 4 pages 73-79). Ici, je vous donne 3 citations pour le justifier

(6) Lesboat people Rohingyas continuent d'affluer en Indonésie et en Thailande en 2009 a été produit par Le Monde. 
«Malgré le refus de la Thaillande et de l'Indonésie de l'accepter, les Rohingyas, groupe ethnique musulman persécuter en Birmanie, continuer de par la mer par le Bangladesh au péril de leur vie.»(Paragraphe 1) (Données 6)

(8) L'enfer sans frontières des Rohingya a été produit par Le Monde en 2010.

«En fait, ils se retrouvent dans les camps misérables où ils survivent entre famine et choléra.» (Paragraphe 1)(Le Monde, Data 8)

(9) Les Rohingya, d'un enfer l'autre a été produit par Le Monde en 2010.

«Fuyant les persécutions en Birmanie, 200.000 membres de cette minorité musulmane ont traversé la frontière avec le Bangladesh et ont troqué une vie de misère pour une autre. "(Paragraphe 1)(Le Monde, Données 9)

Les citations ci-dessus se trouvent dans le premier paragraphe du discours qui décrit la perception des médias en voyant la condition des réfugié. Ils pensent que les Rohingyas ont fait les grandes risques pour survivre à leur vie.

\section{Compréhension du conflit Rohingya par Le Monde}

Le sujet de la compréhension du conflit Rohingya par Le Monde provienne du 7 discours qui ont produit en 2014-2017. Ils ont le même sujet sur la compréhension du conflit Rohingya par Le Monde. Pour le justifier, il y a 9 citations (ils se trouvent dans le chapitre 4 pages $80-85$ ). Ici, je vous donne 2 citations :

(17) Le discours de L'interminable persécution des Rohingya birmans en 2015 a été produit par Le Monde

«L'origine même du nom de Rohingya est controversée, les historiens birmans soutenus que personne n'en avait entendu parler avant les années 1950.» (Paragraphe 1) (Données 17)

(18) L'interminable persécution des Rohingya birmans a été produit par Le Monde en 2015. «Ce sont les Roms de l'Extrême-Orient, réprimés par le régime birman depuis les lustres, traités, au mieux de« Bengalis », au pire de« monstres noirs ». »(Paragraphe 2)(Le Monde, Data 18)

(21) Comprendre la crise des Rohingyas en Birmanie de 2017 a été produit par Le Monde en 2017.

«Quelle est l'origine de cette crise qui dure depuis des dizaines d'années?» (Paragraphe 2)(Le Monde, Donee 21)

Les citations ci-dessus racontent histoire de la venue de Rohingya et le début de l'histoire de la répression par le gouvernement du Myanmar.Le discoursmentionne le Rohingya comme l'homme de bateau appelée par Le Monde international. Dans l'explication, il y a des explications de raisons économiques, idéologiques et politiques d'un point de vue européen.

\section{Analyse de superstructure}

L'analyse schématique montre le résumé de discours. Il comprend l'introduction, le contenu et la clôture.

\section{Analyse schématique de la recherche de protectiondu Bangladesh par Rohingya}

Le premier discours deSur la frontière du Bangladesh Une minorité réprimée raconte l'état actuel de Rohingya. L'introduction de ce titre raconte la condition de la communauté musulmane Rohingya qui a tenté de s'échapper d'Arakan au Bangladesh. Le contenu de ce titre explique la raison de l'évasion 
due à un traitement répressif en Arakan. Ce discours se conclut par l'explication desflux migratoires massifs qui ont eu lieu au Bangladesh.

Le deuxième deBIRMANIE La tension avec le Bangladesh illustre l'isolement de la junteen 1991raconte les efforts du gouvernement bangladais pour tenir une réunion diplomatique avec la Birmanie. L'introduction de ce titre raconte l'implication de Rohingya musulmans rebellesqui ont tué 15 soldats birmans. Ce discours se conclut par l'explication de la situation des Rohingyasest le même quela tribu Karen qui s'était déjà passé au Myanmar.

Le troisième discours de La tension entre Dacca et Rangoun et le Bangladeshmet ses troupes en alerte face à la Birmanie a été produit en 1992. L'introduction de ce titre raconte l'état des Rohingyas au Bangladesh. Le contenu de ce titre raconte la clarification du gouvernement du Myanmar pour justifier de l'assassinat de la société civile Rohingya. Ce discours se conclut par les efforts de prendre des mesures diplomatiques pour résoudre le conflit.

\section{Analyse schématique de la perception du monde internationale sur le conflit Rohingya}

Le discours de «Nettoyage ethnique» en Birmanie chez les musulmans Rohingyasayant été produit en 2000 raconteque le nettoyage ethnique en Birmanie. L'introduction de ce titre raconte le nettoyage ethnique qui se déroulait secrètement au Myanmar. La section de contenu indique à la Fédération des droits de l'homme (FIDH) qui a mené la recherche provenant de l'état deRohingya au Bangladesh. Ce discours se conclut par des critiques de la FIDH envers le HCR concernant le rôle ambigu dans le traitement du conflit Rohingya.

Le discours deLes boat people Rohingyas continuent d'affluer en Indonésie et en Thailande a été produit en 2009. L'introductionde ce titre racontedes Rohingyas qui ont été ignoré par la Thailande et l'Indonésie, ils ont continué à chercher refuge en Thaïlande et en Indonésie par la route maritime du Bangladesh. Le contenu celui raconte la situation d'urgence de Rohingya, le manque de nourriture et de boisson et de mauvaise santé. Ce discours se conclut par la réaction de la Thaïlande qui souhaite participer pour aider à résoudre le conflit Rohingya en faisant appel à l'aide internationale.

Le discours de L'enfer sans frontières des Rohingyas a été produit en 2010.L'introduction de ce titre raconte un récit narratif de la persécution perpétrée contre la minorité musulmane qui a déplacé 200.000 personnes de Birmanie. Le contenu de ce titre montre des conditions de camp impossibles. Ils ont des flambées de choléra dans les camps de réfugiés. Ce discours se conclut par le recit de Rohingya vivant dans la prison de Bangladesh à cause des actescriminels autour du camp de réfugiés.

Le discours de Nouvelles exactions contre les Rohingyas en Birmanie a été produit en 2012. L'introduction de ce titre s'ouvre sur l'exposition du fait de la mort des réfugiés Rohingya donnée par les Nations Unies (ONU). Le contenu de ce titre explique des jugements de Human Rights Watch (HRW) que, selon ses observations, les Rohingyas étaient considérés par le peuple birman comme des occupants de la terre de Birmanie qui harcelaient les bouddhistes. Ce discours se conclutpar la révélation d'une étude de l'ONU qui affirme que le nombre de musulmans Rohingya évacués atteint75.000 habitants.

Le discours deLe président birman demande à l'UE levée des sanctions a été produit en 2013. L'introduction de ce titreraconte la levée des sanctions économiques et politiques qui ont été prises par l'Union européenne. Le contenu de ce titre raconte une demande par le président birman à l'Union européenne de lever une autre sanction internationale sous la forme de sanctions d'armes. La couverture du discours raconte les efforts du président Thein en visitant l'Europe pour faire des efforts diplomatiques pour lever les sanctions.

\section{Analyse schématique de lacompréhension du conflit Rohingya par Le Monde}

Le discours de Birmanie: nouveaux heurts entre bouddhistes et musulmans» a été produit en 2014. L'introduction de ce titre explique l'incident actuel du conflit Rohingya qui a conduit le gouvernement 
de Mandalay à être en alerte pour une sécurité stricte. Le contenu de ce titre a commencé à expliquer la chronologie des événements qui ont tué 2 personnes sur des accusations de viol soulevées par la communauté bouddhiste aux commerçants musulmans. La couverture de ce titre explique les origines explosives du conflit entre les musulmans bouddhistes et Rohingyas depuis 2012.

Le discours deBoat people: pourquoi 2015 n'est pas 1979 a été produit en 2015. L'introduction de ce titre s'ouvre avec une explication de la société européenne aux immigrants qui sont morts en train de se rendre en Europe. Le contenu de ce titre explique la justification des allégations d'ignorance de la communauté européenne. Ce discours se referme sur l'histoire desintellectuels qui ont aidé l'homme de bateau en 1979, mais pour sauver l'homme de bateau deRohingyas maintenant, ils semblent disparaître de la civilisation.

Le discours de Nous refusons le silence face à l'apartheid et au nettoyage ethniquea été produit en 2015. L'introduction de ce titre explique l'appel à toutes les parties pour aider à résoudre le conflit Rohingyas. Le contenu de ce titre confirme que la communauté internationale ne devrait pas fermer les yeux sur l'affaire de nettoyage ethnique des Rohingyas au Myanmar. La couverture de ce titre contient des invitations à continuer de lutter contre l'injustice et l'oppression de Rohingya.

Le discours de Le gouvernement birman accuse de" purification ethnique "a été produit en 2016. L'introduction de ce titre commence avec les récits de l'ONU et internationaux dénonçant la persécution des Rohingyas. Le contenu de ce titre explique la situation du conflit Rohingya qui a été encore exacerbée par la présence de rebelles musulmans dans les efforts du gouvernement Birmanie.Il raconte aussi le critique du monde international à Aung Tsan Tsu Kyiqui ne peut pas resoudre la conflict de Rohingya. La couverture de celui explique le nombre de morts du conflit à Maungdaw a atteint 70 personnes.

Le discours de Comprendre la crise des Rohingyas en Birmanie a été produit en 2017. L'introduction de ce titre explique les estimations de l'ONU selon lesquelles environ 379000 Rohingyas ont quitté le Myanmar depuis août 2016. Le contenu de ce titredécrit l'origine des Rohingyas qui ont conduit à leur existence n'a pas été reconnue au Myanmar. La couverture de ce titre présente la réponse du gouvernement birman à l'opinion selon laquelle la position de Aung Tsan Tsu Kyi est très difficile car elle doit faire face à des extrémistes bouddhistes.

\section{Analyse de microstructure}

L'analyse de la microstructure est une analyse des éléments de discours. Il consiste l'analyse de morphologie, de semantics lexicales, et de cohésion.Il y a 16 discours qui ont été analysés dans cette section (voir le chapitre 4 page 109-170). Ici, je vous donne seulement un exemple d'analyse de microstructure sur le discours de Sur la frontière du Bangladesh Une minorité réprimé qui ont été produit en 1991 par Le Monde.

Table d'analyse de la microstructure sur le discours de Sur la frontière du Bangladesh Une minorité réprimé

\begin{tabular}{|c|c|c|c|c|c|}
\hline No & Publication & Tittre & Élements & Donné & Analyse \\
\hline \multirow[t]{2}{*}{1} & \multirow[t]{2}{*}{ 19.09.1991 } & \multirow[t]{2}{*}{$\begin{array}{l}\text { Sur la } \\
\text { frontière du } \\
\text { Bangladesh } \\
\text { Une } \\
\text { minorité } \\
\text { réprimé }\end{array}$} & \multirow[t]{2}{*}{ Setting } & $\begin{array}{l}\text { Tittre : Sur } \\
\text { la frontière } \\
\text { du } \\
\text { Bangladesh } \\
\text { Une } \\
\text { minorité } \\
\text { réprimée }\end{array}$ & $\begin{array}{l}\text { La frontière du Bangladesh } \\
\text { (location) } \\
\text { Une Minorité (participant, } \\
\text { victim) }\end{array}$ \\
\hline & & & & $\begin{array}{l}\text { Lead :Des } \\
\text { milliers de } \\
\text { musulmans }\end{array}$ & $\begin{array}{l}\text { Arakan, Birmanie (location) } \\
\text { Des milliers de musulmans } \\
\text { (Participants, number) }\end{array}$ \\
\hline
\end{tabular}




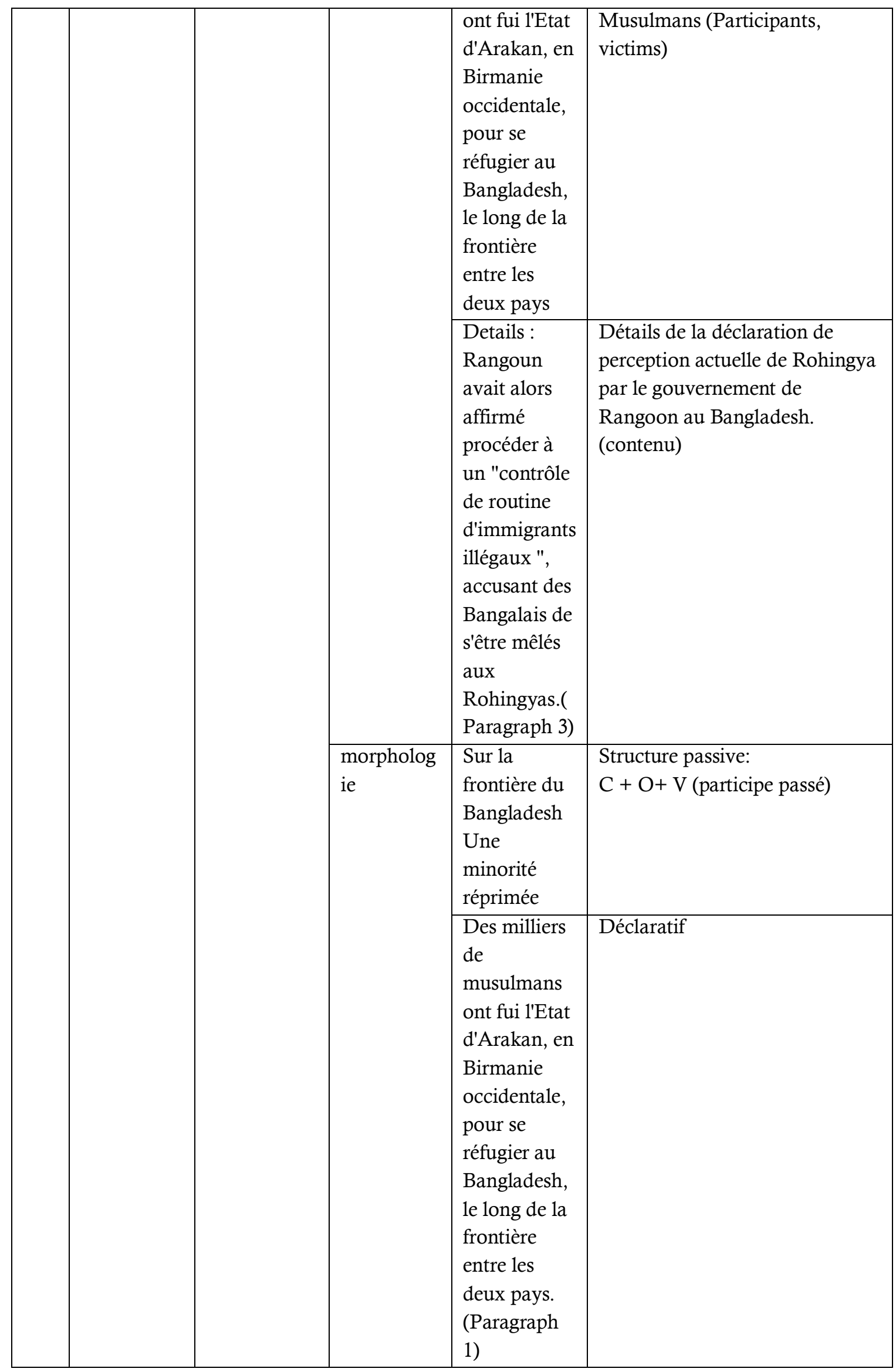




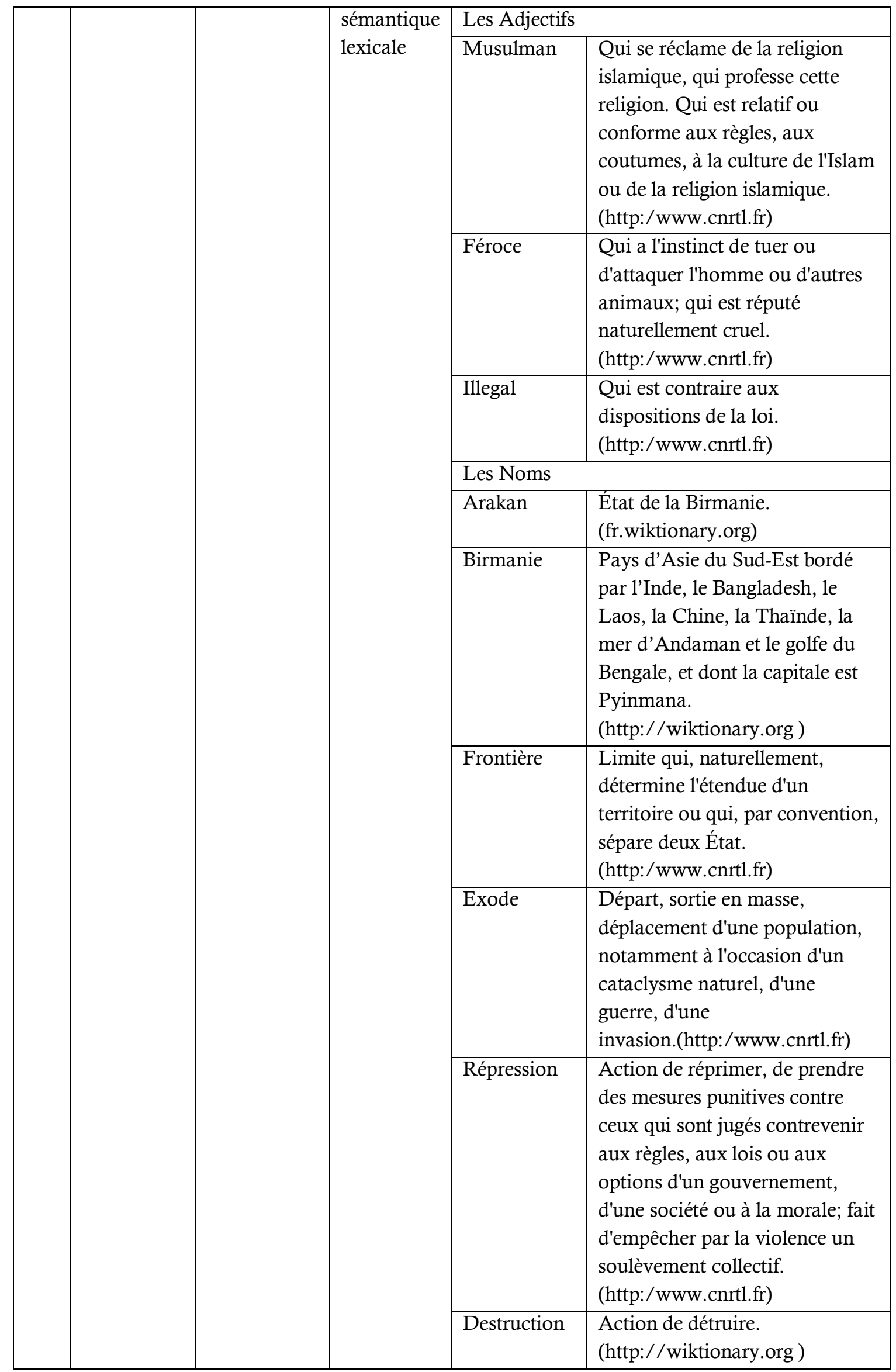




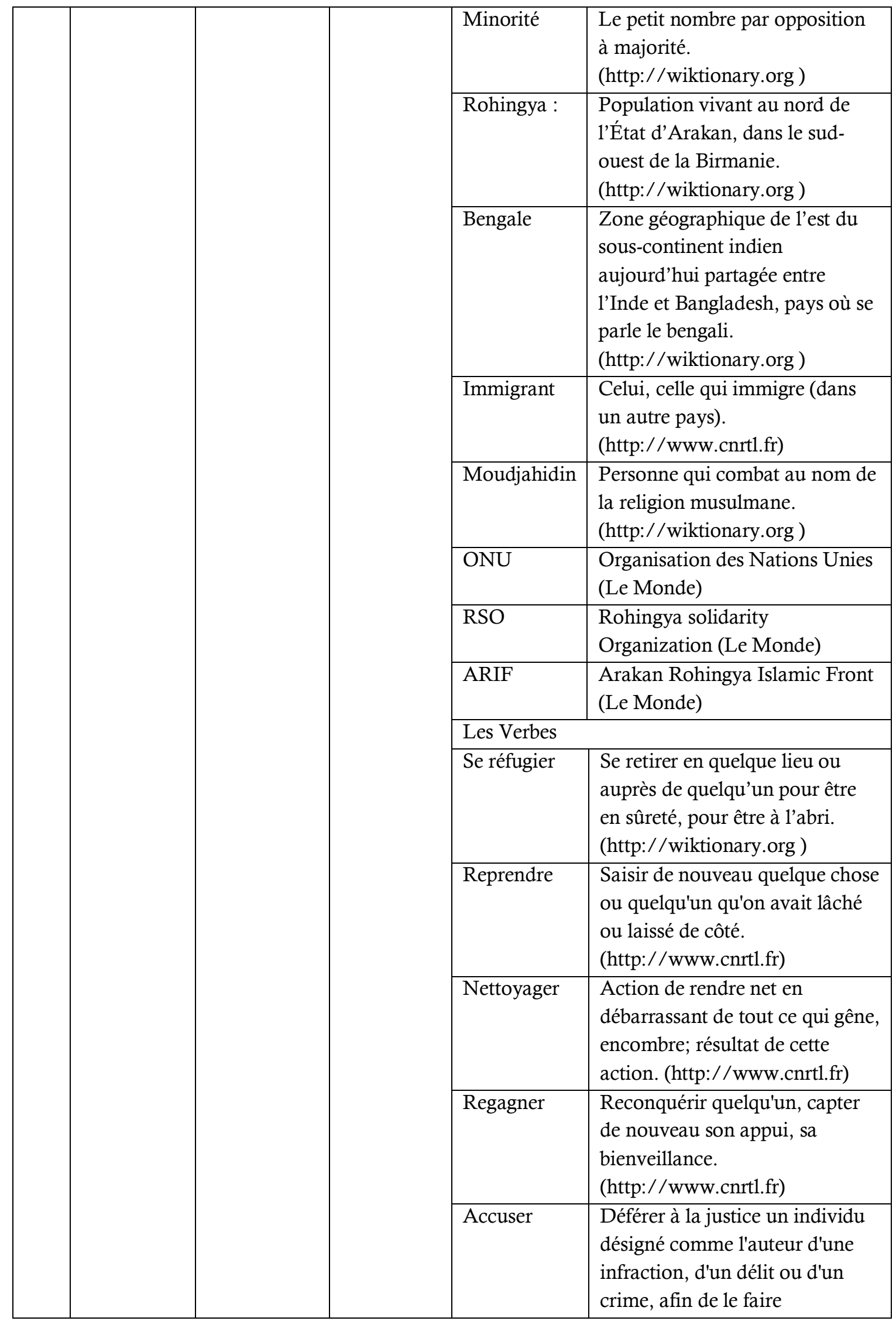




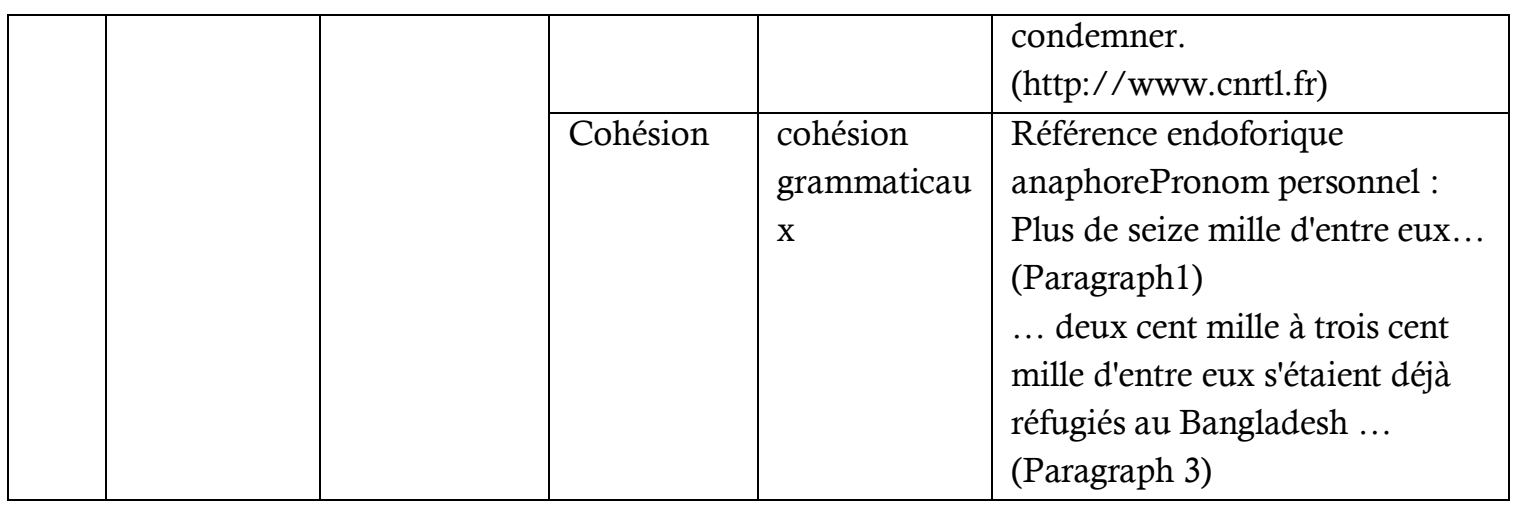

L'analyse de la première étape indiquée dans le tableau montre le schéma d'identification de setting et de participant. Le setting de ce titre a lieu sur la zone frontalière du Bangladesh et du Myanmar. Ensuite, le participant identifié comme victime est un musulman de Rohingya appelé «Musulmans». Dans le premier discours, la classe ennemie n'a pas été clairement identifiée. La mention des musulmans dans le premier discours est un raffinement du mot Rohingya. Une ethnie qui vit un conflit d'oppression au Myanmar.

L'analyse de morphologique décrit l'utilisation des phrases dans le titre du discours. Le discours utilise une structure passive avec la structure de Complément d'objet + Objet + Verbe (Participe passé). Le type de phrase utilisé est une phrase déclarative.

L'analyse de la sémantique lexicale dans le premier discours a trouvé l'utilisation de mots qui ont des nuances politiques. J'analyse tous les mots dans trois categories. Ce sont des adjectifs, des noms et des verbes. Je trouve qu'il y a une répétition des mots à plusieurs reprises dans le discours. Ce sont la répétition du mot «Musulmans» 3 fois, le mot «Rohingya» 4 fois, le mot "Arakan" 4 fois, et le mot "Bangladesh" 4 fois. Dans l'analyse de senses lexicaux, je trouve que le Musulmans ont deux sense de lexicale. Le premier sens, il décrit comme les gens qui se réclament de la religion islamique et professent cette religion. Mais dans le deuxième sens, ils decrivent comme les gens qui sont relatif ou conforme aux règles, aux coutumes, à la culture de l'Islam ou de la religion islamique. Les définitions qui expliquent le sens ont des nuances très différentes. La première définition a des nuances neutres. Mais, la deuxieme définition du sens lexical a un sens négatif au mot «musulman» qui mentionne les musulmans sont ceux qui enfreignent les règles.

\section{Analyse du représentation du Conflit Rohingyasur Le Monde}

Après avoir analysé la macrostructure, la superstructure et la microstructure, j'ai trouvé cinq représentations du journal en ligne Le Monde sur le conflit Rohingya. Ce sont la représentation de la souffrance de l'ethnique Rohingya, de la cruauté du gouvernement du Myanmar, de préoccupations de La FIDH, de L'ONU et de L'UE, la représentationnégative du RSO et de l'ARIF, et la représentation positive de préoccupation Bangladesh.

\section{Représentation de la souffrance ethnique Rohingya}

Analyse de représentation ethnique Rohingya présentant par Le Monde et certains médias internationaux IndiaToday, la BBC et Editorial Media Indonesia illustrent clairement la souffrance ethnique des Rohingyas. Pour justifier cette représentation, il y a 7 citations (ils se trouvent dans la chapitre 4 page 173-176) qui viennent de Le Monde, IndiaToday, et BBC et l'analyse de répetition lexicale et morphologie. Mais ici, j'ai seulement montré une citation.

(23) «Nettoyage ethnique» en birmanie chez les musulmans Rohingyas a été produit en 2000 par Le Monde. 
«Une ÉPURATION ethnique silencieuse est en cours à l'ouest de la Birmanie, dans l'État de l'Arakan: le rapport de la minorité musulmane des Rohingyas, qui continue d'être la victime, dans l'indifférence générale, d'une répression constante de la partie du régime birman.»(Paragraphe 4)(Le Monde, Donnée 4)

(24) L'Enfer sans frontières des Rohingya produit par Le Monde en 2010.

«En fait, ils se retrouvent dans les camps misérables où ils survivent entre famine et choléra.»(Paragraphe 1)(Le Monde, Donnée 8)

Les citations ci-dessus décrivent queles Rohingyas reçoivent un traitement abusif de l'armée de la Junta reçu par les Rohingyas, couvrant le viol des femmes, le massacre des enfants, l'incendie et le pillage des maisons des Rohingyas. La souffrance continue au Bangladesh. Ils sont dans des camps inadaptés, souffrent de malnutrition, de choléra et de maladies de la peau causant la mort de nombreux bébés et enfants.

\section{Représentation de la cruauté du gouvernement du Myanmar}

La cruauté du gouvernement du Myanmar est clairement illustrée par Le Monde et certains médias internationaux de India Today, et $\mathrm{BBC}$. La justification de cette représentation est 12 citations (ils se trouvent dans la chapitre 4 page 182-193). L'une des citations est :

(32) La tension entre Dacca et Rangoun Le Bangladesh a rencontré ses troupes en alerte face à la Birmanie a été produit par Le Monde en 1992.

«Ils fuyaient les violences perpétrées à leur endroit par les troupes de la Birmanie bouddhiste. Rangoun a reconnu que l'action du 21 décembre était une méprise: ses soldats croyaient s'attaquer à un camp d'entraînement de la RSO (organisation de solidarité Rohingya), qui a pris les armes pour une autonomie de l'Arakan. »(Paragraphe 2)(Le Monde, 1992)

La citation ci-dessus explique que l'attaque de la Junte contre le groupe Rohingya Muslim Solidarity a un impact sur les Rohingyas. Le gouvernement birman applique des règles cruelles à Rohingya sous la forme de travail forcé sans salaire et de paiement d'une taxe foncière. Le gouvernement du Myanmar a décrit comme incarné " démon maudit " sous prétexte d'utiliser les Écritures. Ils sont montrés comme les auteurs des opérations de génocide aux Rohingyas ethniques.

\section{Représentation négative du RSO et de l'ARIF}

La mention de RSO (Rohingya Solidarity Organisation) et (ARIF Arakan Rohingya Front islamique) ont une image négative. La justification de cette représentation montre sur l'une citation suivante.

(41) Sur la frontière du Bangladesh Une minorité réprimée a été produit parLe Monde en 1991.

«Les opérations militaires menées ces deux dernières années par l'armée birmane s'expliquent, au moins en partie, par la formation de deux mouvements rebelles musulmans. Le plus important et le plus dur, la RSO (Rohingya solidarity Organization) compterait un demi-millier de moujahidines relativement bien équipés en armes légères et entraînés par des résistants afghans. Le deuxième, plus modéré, l'ARIF (Arakan Rohingya Islamic Front), aurait beaucoup moins d'hommes et moins d'armes. L'existence de ces deux guérillas ne semble pas, cependant, à l'origine de la répression actuelle.» (Paragraph 4) (Le Monde, 1991)

La citations ci-dessus montre le point de vue de Le Monde qui pense que les deux organisations ne sont pas simplement des militants qui luttent mais ils ont d'autres intentions dans le cadre islamique radical. RSO et ARIF ont dépeint comme un réseau terroriste qui ne se limitait pas à résoudre le 
conflit. Les modèles de formation des armes sont armés d'une formation militaire d'Al-Qaïda a conduit à la suspicion de Le Monde.

\section{Représentation de préoccupations de La FIDH, de L'ONU et de L'UE}

L'analyse de représentation de la FIDH, de l'ONU et des préoccupations de l'UE par Le Monde est présentée positivement. Pour justifier cette représentation, il y a 4 citacions (ils se trouvent dans la chapitre 4 page 1991-201). Ici, je vous donnes seulement une citation.

(42) Comprendre la crise des Rohingya en Birmaniea été produit par Le Monde en 2017.

"Le Conseil de sécurité de l'ONU s'est réuni mercredi 13 septembre pour évoquer ce dossier, principal partenaire économique de la Birmanie. A l'issue de cette réunion a huis clos, le Conseil de sécurité a réclamé " des pas immédiats" de la part du gouvernement birman pour faire cesser " une violence excessive. Mardi, a réitéré son "soutien" à la Birmanie et aux "efforts" des autorités birmanes pour "préserver la stabilité» dans l'ouest du pays.» (Paragraph17) (Le Monde, 2017)

Basée sur la citation ci-dessus, L'ONU ou l'Organisation des Nations Unies est très active dans les efforts à resoudre le conflit de Rohingya.

\section{Représentation positive de préoccupation Bangladesh}

L'analyse de la représentation du Bangladesh selonLe Monde est représentée positivement en raison de son souci de recevoir les réfugiés Rohingyaet jouer un rôle actif dans l'effort de résoudre des conflits Rohingya. Pour justifier cette représentation, il y a 5 citations (il se trouvent dans la chapitre 4 page 205-206).

(47) La tension entre Dacca et Rangoun Le Bangladesh met ses troupes en alerte face à la Birmanie tahun a été produit parLe Monde en 1992.

"Le Bangladesh et la Birmanie devaient entamer, mardi 31 décembre, dans la ville birmane de Maungdaw, des pourparlers à la suite de récents accrochages frontaliers qui ont provoqué une mobilisation militaire dans les deux pays.» (Paragraph 1)(Le Monde, 1992)

La citation ci-dessus montré que le gouvernement de Bangladesh mène l'investigation sur les personnes bangladaises qui dérangent les Rohingyas et invite activement la communauté internationale à participer à l'aide au règlement des conflits par la consolidation sur les voies diplomatiques. Une autre chose est réellement montré dans IndiaToday comportant une mauvaise image du Bangladesh qui fournit un bidonville et un camp de refuge inadéquat et ne fournit pas une nourriture adéquate à ceux qui souffrent de malnutrition. Les reportages négatifs d'Indiatoday sur le Bangladesh ont un élément politique d'un point de vue historique. Historiquement, le Bangladesh a été formé par la guerre et le conflit Inde-Pakistan qui étaient des guerres et des conflits entre l'Inde et le Pakistan, depuis la séparation de l'Inde en août 1947.

Une image positive est également affichée par Editorial Media Indonesia, qui fait partie de Metro TV qui appartenu à Surya Paloh du Parti Nasdem. La coalition de Surya Paloh avec le gouvernement de Joko Widodo, est devenue l'un des médias rapportant activement les activités politiques menées par le gouvernement de Joko Widodo.

Basée sur l'analyse ci-dessus, il est clair que la représentation de tous les médias qui prêchent un événement devient une tendance et être partisane envers certains partis. En outre, les éléments idéologiques et la puissance de chaque nouvelle sont une inséparable du processus de production de nouvelles. 


\section{LA CONCLUSION}

Basé sur les résultats de la recherche au dessus, j'ai conclu 5 points très importants liées à la partisanerie de Le Monde sur la conflit Rohingya.

Premièrement, Le Monde s'est rangé du côté des Rohingyas ethniques. L'alignement est conformé à l'idéologie adoptée par Le Monde qui défend la liberté et la protection des droits de l'homme. Le Monde montre de l'empathie pour les violations des droits de l'homme commises contre les Rohingyas au Myanmar. L'alignement de Le Monde est attesté parl'identification du setting, du lexique et de la morphologie. Dans le setting du discours, Rohingya est toujours décrit comme une victime (la partie lésée).Ensuite, le mot "Rohingya" dans la période 1991-2017 est mentionnée 101 fois.En outre, en termes de morphologie, Le Monde parle jusqu'à 6 fois en utilisant une forme de nominalisation qui décrit l'état de souffrance de Rohingyaet la forme pssive est mentionné 2 fois montrant la projection de la victime.La souffrance des Rohingyas est décrite en utilisant la parabole du mot «Neraka» qui signifie «enfer».

Deuxièmement, Le Monde s'est rangé du côté du Bangladesh en présentant une image positive des préoccupations du Bangladesh dans ses efforts pour résoudre au conflit Rohingyas. Cet alignement semble très différent avec les représentations d'India Today qui tendent à représenter une image négative. Ces différences se produisent en raison de facteurs idéologiques et historiques. Idéologiquement, Le Monde voit les actions du Bangladesh en accord avec les principes humanitaires et les efforts de résolution des conflits internationaux. Le Bangladesh dans la perspective de Le Monde a joué un rôle approprié dans la préparation de l'aide à l'accueil des réfugiés Rohingya au Bangladesh et dans la résolution des conflits par la diplomatie en négociant avec les pays de l'ASEAN et l'Organisation Internationale (UE, ONU). La justification de l'alignement de Le Monde est vue dans le rapport d'identification contexte montrant le Bangladesh comme victime du conflit de Rohingyas qui doit accepter l'impact d'une migration massive de Rohingyas au Bangladesh. Pendant ce temps, India Today, en tant que journal du gouvernement indien impliqué dans la guerre d'indépendance du Bangladesh en 1971, a encore des tensions politiques et sociales contre le Bangladesh qui a engagé une guerre contre l'Inde.

Troisièmement, Le Monde s'est rangé du côté du Bangladesh en présentant une image positivedu FIDH (Fédération internationale des ligues des droits de l'homme), ONU (Organisation des Nations Unies) et EU (European Union).Des alignements similaires sont également montrés par la BBC.Toutes les deux médias montrent l'activité de la FIDH et de l'ONU en donnant aux victimes des informations et des rapports, sans compter que l'ONU est également très préoccupée par la vie des réfugiés Rohingya en envoyant des diverses aides humanitaires par l'intermédiaire du HCR. La justification de la description est évidente dans la citation" Efforts to resolve the issue must focus on "the root cause" inside Myanmar, Mr McKissick, head of the UN refugee agency UNHCR» dan «Le Conseil de sécurité de l'ONU s'est réuni mercredi 13 septembre pour évoquer ce dossier, principal partenaire économique de la Birmanie ...».

Quatrièmement, Le Monde ne s'est rangé pas du côté du gouvernement du Myanmar. La même chose est montrée par IndiaToday, BBC et Editorial Media Indonesia. Les quatre médias décrivent simultanément les atrocités du gouvernement du Myanmar pour les actes répressifs contre les Rohingyas. Justification de la représentation se voit dans l'utilisation de phrases actives autant que 7 fois de 16 titres de discours. Les sept titres mettent clairement en évidence les auteurs d'une action répressive (le gouvernement du Myanmar). En outre, sur Le Monde, on utilise le «nettoyage», «Purge» sur IndiaToday, et «Purification» sur la BBC, ce qui signifie «nettoyage» désigne les actions génocidaires ainsi que le massacre ethnique Rohingya à Myanamr.

Cinquièmement, Le Monde dénote implicitement la partialité envers le RSO (Rohingya Solidarity Organization) et l'ARIF (Arakan Rohingya Islamic Front). L'existence de deux organisations n'est pas considérée comme une organisation militante qui défend le droit de la vie des 
Rohingyas, mais est considérée comme un groupe islamique radical qui saisit l'opportunité du conflit Rohingyas pour propager le radicalisme. Il est illustré par la justification de la citation «L'existence de ces deux guérillas ne semble pas, cependant, à l'origine de la répression actuelle». Indirectement la citation signifie que les deux organisations (RSO et ARIF) ont d'autres intentions pour leur existence en tant qu'organisation qui défend les Rohingyas, ils ne sont pas simplement des militants qui luttent mais plutôt l'explication qu'ils ont d'autres intentions dans un cadre islamique radical. Ceci est encore renforcé par une déclaration confirmant que le RSO reçoit des armes des réseaux terroristes afghans appartenant à Al-Qaïda. Ce point de vue rejoint la vision de la mission de Le Monde en tant que média officiel du gouvernement français fermement opposé aux terroristes.

\section{LA BIBLIOGRAPHIE}

Abelson, R. P. 1976. Cognition and Social. Behaviour. New York: Earl Baun.

Ahmad Bilal, H. et al. 2012. Critical Discourse Analysis of Political TV Talk Shows of Pakistani Media'.International Journal of Linguistics. Vol 4. No 3: 203-219. Las Vegas: Macrothink Institute.

Amnesty International. 2015. Open Letter: South Asia Refugee Crisis. London: Amnesty International International Secretariat.

Archer, Clive. 1984. International Organization. London: University of Aberdee.

Bakk. 2011. The Critical Analysis of Style: The Case of Press Releases. Wien: Universtät Wien.

Barry, Peter. 2010. Beginning Theory. Yogyakarta: Jalasutra.

Berutu, Tua Hendra. 2010. Penerapan Pendekatan Analisis Wacana Kritis Model Teun A. van Dijk dalam Meningkatkan Kemampuan Membaca Pemahaman Teks Pada Surat Kabar Perancis (Le Monde). Skripsi. Universitas Negeri Yogyakarta.

Billig. 2013. The Language of Critical Discourse Analysis: The Case of Nominalization. Loughborough: Loughborough Universty.

Bocquet, B. 2016. Dominant Ideology and Racism in the French Media : A Critical Discourse Analysis on the Case of the Denaturalization Law. Journal of Political Science. Vol 6. No 1: 54-69. Uppsala: Uppsala University.

Brough, J.B., 2009. Edmund Husserl 1859-1938: In Handbook of Phenomenological Aesthetics. Dordrecht: Springer.

Brown, Gillian dan Yule., George. 1996. Analisis Wacana. Jakarta: PT. Gramedia Pustaka Utama.

Budianta, Melani dkk. 2002. Membaca Sastra: Pengantar Memahami Sastra untuk Perguruan Tinggi. Depok: Indonesiatera.

Cherlet, T. 2015. Analyse Critique Du Discours (Critical Discourse Analysis) de Textes Journalistiques Sur La Crise Migratoire Dans Les Journaux Belges. Gent: Universiteit Gent.

Cook, Guy. 1994. The Discourse of Advertising.London and New York: Routledge.

Culler, Jonathan. 1981. Structuralist Poetics. London: Routletge \& Kegan.

Darma, Yoce Aliah. 2014. Analisis Wacana Kritis. Bandung: Yayasan Widya.

Dubois. 2001. Dictionnaire de la Linguistique. Paris: Larousse-Bordas.

Ekodhanto, Frans. 2009. Analisis Wacana Kritis Ideologi Politik Media Massa Mada Teks Tajuk Terencana Kompas dan Pikiran Rakyat. Skripsi. Universitas Indonesia.

Eldin, A. A. T. S. 2014. Critical Discourse Analysis of Religious Sermons In Egypt-Case Study of Amr Khalid's Sermons. International Education Studies. Vol 7. No 11: 68-75. North York: Canadian Center of Science and Education.

Eriyanto. 2001. Analisis Wacana: Pengantar Analisis Teks Media.Yogyakarta: PT. LKIS Pelangi Aksara. 2006. Analisis Wacana: Pengantar Analisis Teks Media.Yogyakarta: PT. LKIS Pelangi Aksara.

Fairclough, Norman. 1995. Critical Discourse Analysis. New York: Longman. 2001. Language and Power. England: Pearson Educated Limited.

Faruk. 2008. Pengantar Sosiologi Sastra dari Strukturalisme Genetik sampai Post-Modernisme. Yogyakarta: Pustaka Pelajar.

Fiske, John. 2011. Cultural and Communication Studies. Yogyakarta: Jalasutra.

Foucault, M. 1997. Ethics: Subjectivity and Truth. New York: New Press.

Fowler, R. 1997. Literature as discours. Bloomington: Indiana University Press. 
Ghannam. 2011. A Critical Discourse Analysis of an Event Published in Six Lebanese Newspape. Witwatersrand: University of the Witwatersrand

Gray, J., 2006. Watching with The Simpsons: Television, Parody, and Intertextuality. London: Taylor \& Francis.

Halliday, M.A.K. 1978. Language as Social Semiotic: The Social Interpretation of Language and Meaning. London: Edward Arnold.

Harris, S. Zellig. 1952. Discourse Analysis. Journal of Languistic. Vol. 28. No. 1: 1-30. New York: Linguistic Society of America

Hawthorn, J. 1992. A Concise Glossary of Contemporary Literary Theory. London: Edward Arnold.

Hutomo, S.S. 1993. Merambah Matahari: Sastra dalam Perbandingan. Surabaya: Gaya Masa.

Images, Asia. 1997. Report On The Situation For Muslims In Burma. Bangkok: Forma Asia.

J.S. Badudu, 2000. Kamus Umum Bahasa Indonesia. Jakarta: Pustaka Sinar Harapan.

Joko Santoso, B. Wahyudi. 2018. Representasi Aktor Sosial Politik dan Pertarungan Makna Dalam Pemilihan Umum Kepala Daerah (PILKADA) Tingkat Provinsi di Pulai Jawa Pada Tahun 2018 di Media Massa Daring Dalam Perspektif Analisis Wacana Kritis Model Theo van Leeuwen.Kolita: Konferensi Linguistik Tahunan Atma Jaya. ISSN: 2549-810X. Jakarta: Universitas Katolik Indonesia Atma Jaya

Jorgensen, M.W. and Phillips, L.J., 2007. Analisis Wacana: Teori dan Metode. Yogyakarta: Pustaka Pelajar.

Junus, Umar. 1985. Resepsi Sastra: Sebuah Pengantar.Jakarta: Gramedia.

Keraf, Gorys. 1997. Komposisi. Jakarta: Ikrar Mandiri Abadi.

Laclau, E. and Mouffe, C. (1985) Hegemony and Socialist Strategy. Towards a Radical Democratic Politics. London: Verso.

Miles, M.B. and Huberman, A.M., 1984. Qualitative Data Analysis: An Expanded Sourcebook. London: Sage Publications.

Mills, S. 1997. Discourse. London: Routledge.

Muhadjir, Noeng. 1996. Metodologi Penelitian Kualitatif. Yogyakarta: Rake.

Plano, Jack C. dan Roy Olton. 1999. Kamus Hubungan Internasional. Translated by Drs. Wawan Juanda. Bandung: PT Putra A. Bardin.

Rahimi, F. and Riasati, M. 2011. Critical Discourse Dnalysis: Scrutinizing Ideologically-Driven Discourses. International Journal of Humanities and Social. Vol 1. No 16: 107-112. New York: Center for Promoting Ideas.

Ramanathan, R. and Hoon, T. 2015. Application of Critical Discourse Analysis in Media Discourse Studies. Southeast Asian Journal of English Language. Vol 21. No 2: 57-68. Selangor: UKM Press.

Rashidi, N. and Souzandehfar, M. 2010. A Critical Discourse Analysis of the Debates Between Republicans and Democrats Over the Continuation of War in Iraq. The Journal of Linguistic and Intercultural Education. Vol: 3. No 2: 55-82. Warsawa: Index Copernicus International.

Ratna, Nyoman Kutha. 2011. Teori, Metode, dan Teknik Penelitian Sastra dari. Strkturalisme Hingga Postrukturalisme Perspektif Wacana Naratif.Yogyakarta: Pustaka Pelajar.

Riffaterre, Michael. 1978. Semiotics of Poetry. Bloomington and London: Indiana Unversity Press.

S. Hikam, Muhammad A.1999. Demokrasi dan Civil Society. Jakarta: Pustaka.

Sarup, Madan. 2011.Panduan Pengantar untuk Memahami Poststukturalisme dan Postmodernisme. Yogyakarta: Jalasutra.

Setiadi, Riswanda. 2010. Analisis dan Interpretasi Data Melalui Pendekatan Kuantitatif dan Kualitatif. Bandung: Universitas Pendidikan Indonesia.

Sobur, Alex. 2009. Analisis Teks Media. Bandung: Remaja Rosdakarya.

Sudaryanto. 1993. Metode dan Aneka Teknik Analisis Bahasa. Yogyakarta: Duta.

Sugiyono. 2009. Metode Penelitian Kuantitatif, Kualitatif dan R\&D. Bandung: Afabeta.

Tahmasbi, S. and Kalkhajeh, S. G. 2013. Critical Discourse Analysis : Iranian Banks Advertisements Soheila Tahmasbi Sasan Ghorgani Kalkhajeh Research Questions And Hypotheses. Journal of Asian Economic and Social Society. Vol 3. No 1: 124-145. NewYork: Asian Economic and Social Society.

Teeuw, A. 1980. Sastra Baru Indonesia. Flores: Nusa Indah. Universitas Lampung.

Titscher, Stefan., Michael Meyer., Ruth Wodak, and Eva Vetter. 2000. Methods of Text and Discourse Analysis. London: Sage Publications

Utami, T. B. 2014. Analisis Wacana Kritis Teks Jurnalistik pada Surat Kabar Online Le Monde. Skripsi. Universitas Pendidikan Indonesia. 
van Dijk, Teun A. 1988a. News Analysis: Case Studies of International and National News in the Press. New Jersey: Lawrence Erlbaum Associates.

1988b. News as Discourse. New Jersey: Lawrence Erlbaum Associates.

1991. Racism and the Press. London, New York: Routledge.

2008. Discourse and Context: A Sociocognitive Approach. Cambridge: Cambridge University Press.

2009. Society and Discourse: How Social Contexts Influence Texts and Talk. Cambridge: Cambridge University Press.

van Leeuwen, Theo. 2008. Discourse and Practice. Oxford: Oxford University.

Wang, J. 2010. A Critical Discourse Analysis of Barack Obama's Speeches. Journal of Language Teaching and Research. Vol 1. No 3: 254-261. London: SAGE Publication.

Wenden, A. 2005. The Politics of Representation: A Critical Discourse Analysis of an Aljazeera Special Report. International Journal of Peace Studies. Vol 10. No 2: 89-112. Leuven: International Peace Research Association.

Wittgenstein, L., 1984. Culture and Value. Chicago: University of Chicago Press.

Wodak, R. dan N. Fairclough. 1989. Critical Discourse Analysis. London: SAGE Publication. Anonim. 1991. Birmanie La tension avec le Bangladesh illustre l'isolement de la junte. Diunduh dari http://www.lemonde.fr/international/article/2017/10/20/jacques-leider-la-junte-birmane-ainstrumentalise-le-ressentiment-envers-les-Rohingya_5203777_3210.html [7/10/2017]. ---. 1991. Sur la frontière $d u$ Bangladesh Une minorité réprimée. Diunduh dari http://www.lemonde.fr/archives/article/1991/09/19/sur-la-frontiere-du-Bangladesh-une-minoritereprimee_4033481_1819218.html\#HjPRZduG56hY7gvd.99 [7/10/2017].

- 1992. La tension entre Dacca et Rangoun Le Bangladesh met ses troupes en alerte face à la Birmanie. Diunduh dari http://www.lemonde.fr/archives/article/1991/09/19/sur-la-frontiere-du-Bangladesh-uneminorite-reprimee_4033481_1819218.html\#HjPRZduG56hY7gvd.99 [7/10/2017].

---------. 2010. L'enfer sans frontières des Rohingya. Diunduh dari http://lemonde.fr/a-laune/article/2010/09/22/1-enfer-sans-frontieres-des-Rohingya_1414589_3208.html [7/10/2017].

--. 2013. Le président birman demande à l'UE la levée des sanc. Diunduh dari http://www.lemonde.fr/asiepacifique/article/2013/03/04/le-president-birman-demande-a-l-ue-la-levee-dessanctions_1842575_3216.html [7/10/2017].

--------. 2013. Violences religieuses en Birmanie : des musulmans condamnés. Diunduh dari http://www.lemonde.fr/asie-pacifique/article/2013/05/21/violences-religieuses-en-birmanie-desmusulmans-condamnes_3412275_3216.html [7/10/2017].

--1-- 2014. Birmanie : nouveaux heurts entre bouddhistes et musulmans. Diunduh dari http://www.lemonde.fr/asie-pacifique/article/2014/07/03/birmanie-nouveaux-heurts-entrebouddhistes-et-musulmans_4449816_3216.html [7/10/2017].

---------. 2016. Myanmar wants ethnic cleansing of Rohingya - UN official. Diunduh dari http://www.bbc.com/news/world-asia-38091816 [14/12/2017].

---a----. 2018. History of Myanmar. Diunduh dari https://en.wikipedia.org/wiki/History_of_Myanmar [15/03/2018].

-------. 2018. Media of Myanmar. Diunduh dari https://en.wikipedia.org/wiki/Media_of_Myanmar [15/03/2018].

----------. 2018. Politics of Myanmar. Diunduh dari https://en.wikipedia.org/wiki/Politics_of_Myanmar [15/03/2018].

2018. Union Solidarity and Development Party. Diunduh dari https://en.wikipedia.org/wiki/Union_Solidarity_and_Development_Party [15/03/2018].

Biswas, Soutik. 1992. Purge of the Muslims. Diunduh dari https://www.indiatoday.in/magazine/31-12-1992 [14/12/2017].

Bouissou, Julien. 2010. Les Rohingya, d'un enfer l'autre. Diunduh dari http://www.lemonde.fr/asiepacifique/article/2010/09/22/les-Rohingya-d-un-enfer-1-autre_1414658_3216.html [7/10/2017].

Centre National de Ressources Textuelles et Lexicales. 2012. Dictionnaire. Diunduh dari http://www.cnrtl.fr/definition [14/11/2017].

Geoffroy, Romain. 2017. Comprendre la crise des Rohingya en Birmanie. Diunduh dari http://www.lemonde.fr/lesdecodeurs/article/2017/09/13/tout-comprendre-a-la-crise-des-Rohingya-enbirmanie_5185226_4355770.html\#5iLYe0I0kKZuuiFJ.99 [7/10/2017]. 
Joko Santoso, B. Wahyudi, dan Gallant Karunia Asidik. 2016. Citra Publik Presiden pada Pemberitaan di Harian Suara Merdeka, Tabloid Tempo, dan Harian Rebpublika: Kajian Analisis Wacana Kritis Model Norman Fairclough.Seloka: Jurnal Pendidikan Bahasa dan Sastra Indonesia. Vol 5. No 2: 201-215. Semarang: Universitas Negeri Semarang. Diunduh dari http://journal.unnes.ac.id/sju/index.php/seloka [10/4/2018]

---------, dan Siti Nur Fadhilah. 2017. Pencitraan Soeharto dalam Buku Andai Pak Harto Nyapres, Kupilih! (Kebosanan Orang-Orang Pinggiran Menanti Kemakmuran) dalam Perspektif Analisis Wacana Kritis van Dijk.Seloka: Jurnal Pendidikan Bahasa dan Sastra Indonesia. Vol 6. No 2: 139-151. Semarang: UniversitasNegeri Semarang. Diunduh darihttp://journal.unnes.ac.id/sju/index.php/seloka [19/4/2018]

Jonathan. 2017. Rohingya crisis: Seeing through the official story in Myanmar. Diunduh dari http://www.bbc.com/news/world-asia-41222210 [14/12/2017].

Kauffmann, Sylvie. 2009. Les boat people Rohingyas continuent d'affluer en Indonésie et en Thailande. Diunduh dari http://www.lemonde.fr/asie-pacifique/article/2009/02/03/les-boat-people-Rohingyas-continuent-daffluer-en-indonesie-et-en-thailande_1150028_3216.html [7/10/2017].

-2014. Boat people : pourquoi 2015 n'est pas 1979. Diunduh dari http://www.lemonde.fr/idees/article/2015/05/16/boat-people-pourquoi-2015-n-est-pas1979_4634696_3232.html\#PmrPZBwJdqPGWBY1.99 [7/10/2017].

Larousse. Dictionnaires de Français. Diunduh dari http://www.larousse.fr/dictionnaires/francais/ [4/12/2017].

Le-Dictionnaire. Diunduh dari http://le-dictionnaire.com/ [1/12/2017].

Michaella, Sonya. 2018. Indonesia Siap Bantu Proses Relokasi Etnis Rohingya. Diunduh dari http://internasional.metrotvnews.com/asia/ZkeQG27k-indonesia-siap-bantu-proses-relokasi-etnisRohingya [19/03/2018].

Philip, Bruno. 2000. Nettoyage ethnique en Birmanie chez les musulmans Rohingyas. Diunduh dari http://www.lemonde.fr/asie-pacifique/article/2017/09/20/le-Bangladesh-demande-a-la-birmanie-dereadmettre-les-refugies-Rohingya_5188215_3216.html [7/10/2017].

--------. 2012. L'interminable persécution des Rohingya birmans. Diunduh dari http://www.lemonde.fr/asiepacifique/article/2012/06/21/1-interminable-persecution-des-Rohingyasbirmans_1722542_3216.html\#mWIv7i2b7utG8pgV.99 [7/10/2017].

. 2012. Nouvelles exactions contre les Rohingya en Birmanie. Diunduh dari http://www.lemonde.fr/asiepacifique/article/2012/10/29/nouvelles-exactions-contre-les-Rohingya-enbirmanie_1782605_3216.html [7/10/2017].

- 2014. A Sittwe, pour les habitants, les Rohingya "n'existent pas". Diunduh dari http://www.lemonde.fr/asiepacifique/article/2014/11/25/a-sittwe-le-drame-invisible-des-Rohingya-peuple-qui-n-existepas_4529023_3216.html [7/10/2017].

---------. 2016. Le gouvernement birman accusé d 'purification ethnique'. Diunduh dari http://www.lemonde.fr/asiepacifique/article/2016/11/25/le-gouvernement-birman-accuse-de-purificationethnique_5037823_3216.html [7/10/2017].

Wiktionary. 2017. Diunduh dari https://fr.wiktionary.org/wiki/ [27/12/2017].

Zarni, Maung 2013. Nous refusons le silence face à l'Apartheid et au nettoyage ethnique en Birmanie. Diunduh dari http://www.lemonde.fr/idees/article/2013/06/26/nous-refusons-le-silence-face-a-1-apartheid-et-aunettoyage-ethnique-en-birmanie_3436755_3232.html\#xDJ8Xw68GPefcZd8.99 [7/10/2017] 